\title{
An interpretative structural model based on SPSS for the interaction analysis between risk factors of power grid
}

\author{
Yichao Huang ${ }^{1}$, Ruanming Huang ${ }^{1}$, Xinjian $\mathrm{Li}^{2}$, Shujuan Chen ${ }^{3}$, Mingxing Guo ${ }^{3}$, Qinjiang ${ }^{3}$, Yuqing $\mathrm{He}^{4 *}$ \\ ${ }^{1}$ Economic and Technological Research Institute of Shanghai Power Grid, State Grid Shanghai Electric Power Company, 200122, \\ Shanghai, China. \\ ${ }^{2}$ Energy Development Research Institute, Hunan University, State Grid Yiyang Electric Power Company Company, 413002, Yiyang, \\ China \\ ${ }^{3}$ Key Laboratory of Intelligent Information Analysis and Comprehensive Optimization of Energy Internet in Hunan Province, Hunan \\ University, 410082, Changsha, China \\ ${ }^{4}$ Economic and Technological Research Institute of Hunan Power Grid, State Grid Hunan Power Co., Ltd, 410007, Changsha, China
}

\begin{abstract}
The risk factors influencing the analysis of power grid investment are complicated and highly coupled. The analysis of the interaction of risk factors based on the traditional interpretative structural model, using the expert score to form correlation matrix, which is subjective. To increase the quantitative analysis and overcome the drawbacks of traditional model relying on experts' experience, this paper proposes a new method to construct the interpretative structural model. Firstly, the evaluation index system of power grid investment is constructed, based on this, the collected history data of risk factors are processed and analysed through the correlation analysis of SPSS and the quantitative correlation matrix of risk factors is obtained, then the construction of interpretative structural model is finished. The constructed model provides the basis for the analysis and management of power grid investment.
\end{abstract}

\section{Introduction}

With the insert of large distributed power, the use and spread of smart grid, the risk management of grid power faces a large challenge [1]. On the one hand, compared with the single power of traditional energy like thermal power, hydro power and nuclear power, the renewable clean energy like wind power, solar power, tide power have been switched in power grid in user's end, which makes the risk factors influencing the safety of power grid increase and harder to forecast [2], on the other hand, risk factors influence each other, with the increase of risk factors, the possibility of risk factors influencing each other and forming coupling risk is increasing. When it occurs, it is more difficult to control and prevent risk, moreover, the management of power grid becomes harder and may bring inestimable loss. Therefore, the analysis of the phenomenon of risk coupling is significant in power grid [3].

Risk coupling is the phenomenon that two or more risk factors influence each other through all kinds of interactions in the risk chain [4]. The phenomenon of interactions like offset and expansion may occur and result in the change of risk size which deviate from the expectation and cause loss. In the traditional interpretative structural model [5], the method of expert scoring is applied to consider the interaction of risk factors, but the description of interaction between risk factors only have two values, 0 and 1,0 means there is no coupling between risk factors and 1 means there is coupling between risk factors, ignoring the strength of risk factors. The literature [6] proposes an improved interpretative structural model to analyze the renewable energy program, which uses the expert scoring to get the strength of interaction between risk factors but ignoring the quantitative analysis and not being objective enough.

In this paper, to avoid the expert scoring's objective analysis, an interpretative structural model based on SPSS for the interaction analysis between risk factors of power grid is proposed. The process and analysis of history risk data are finished, to achieve the quantitative analysis between risk factors.

\section{Construction of risk index system}

There are lots of risk factors influencing the power grid investment, in order to make sure the comprehensiveness and objectivity of the selection of indexes, in this paper, firstly, according to the investigation, 28 uncertain factors that may exist in the power grid investment are recognized, based on this, the fuzzy theory [7] is applied to recognize the $12 \mathrm{key}$ uncertain factors, and then the classification of factors is finished according to the characteristic of uncertain factors. The BP neural network model based on genetic algorithms [8,9] is applied to finish the filtration of key uncertain factors and recognize the 10 factors that may turn into risk,

$\overline{{ }^{*} \text { Corresponding author's e-mail: iheyuqing@163.com }}$ 
which makes sure the selection of risk factors is feasible. Finally the established risk index system is listed as Table. 1 .

Table 1. Risk index system.

\begin{tabular}{cc}
\hline First grade risk & Second grade risk \\
\hline Policy risk A & electricity purchasing cost A1 \\
& average power price A2 \\
& new energy policy A3 \\
load demand B1 & GDP growth B2 \\
Economic & loan interest rate B3 \\
development risk B & power supply capacity C1 \\
& grid structure C2 \\
Power grid & technological levelC3 \\
development risk C & power quality C4 \\
\hline
\end{tabular}

\section{Analysis of risk coupling}

Based on the established risk index system, according to the process of the collected history data, the error of risk factor is obtained. The error of risk factor between actual value and predicated value results in the risk value of uncertain factor. Through the process of the collected history data, the predicted value of history data are acquired. The data processing means establishing the Autoregressive Moving Average Model (ARIMA), and the actual history data before the predicted data needed in present year are as the input of ARIMA.

The formula of calculating the correlation coefficient [10] between each two risk factors is as follows.

$$
r=\frac{\sum\left(x_{i}-\bar{x}\right)\left(y_{i}-\bar{y}\right)}{\sqrt{\sum\left(\left(x_{i}-\bar{x}\right)\right)^{2}} \sqrt{\sum\left(\left(y_{i}-\bar{y}\right)\right)^{2}}}
$$

Where $r$ is the strength of correlation between each two risk factors, which ranges from -1 to $1 . x_{i}$ and $y_{i}$ are the $i$ th sample value of two variables, $x_{i}$ and $y_{i}$ are the average value of $x$ and $y$ respectively.

\section{Construction of interpretative structural model}

Through the correlation matrix of risk factors, the construction of interpretative structural model is finished, the processes of constructing the hierarchical structure model are as follows.

\subsection{Getting the correlation strength matrix B}

Based on the selected membership function [11] and the calculated correlation matrix, the calculation of the correlation strength matrix is as follows.

$$
b_{i j}=r_{i j} /\left(r_{i .}+r_{. j}-r_{i j}\right)
$$

Where $r_{i}$ is the sum of the $i$ th row elements in the matrix and $r_{. j}$ is sum of the $j$ th column elements in the matrix.

\subsection{Forming the adjacent matrix $A$}

After the process of correlation strength matrix, the adjacent matrix $A=\left(a_{i j}\right)_{n \times n}$ of the selected threshold is calculated as follows.

$$
a_{i j}= \begin{cases}1 & b_{i j} \geq \lambda \\ 0 & b_{i j}<\lambda\end{cases}
$$

Where $a_{i j}$ is the element of adjacent matrix $A, b_{i j}$ is the element of correlation strength matrix, $\lambda$ is the threshold, $n$ is the order of adjacent matrix.

In general, the threshold is smaller, the partition of system is thicker, while the threshold is bigger, and the partition of system is thinner. In the research of literature [12], the whole property is the best under the threshold between 0.05 and 0.08 .

\subsection{Calculating the reachable matrix}

Through the operation of adjacent matrix, the reachable matrix is calculated. The reachable matrix reflects the direct and indirect relations of factors.

Confirming reachable set $S i$ and leading set $B i$ and calculating the intersection of $S i$ and $B i$. If there is $R\left(S_{i}\right) \cap A\left(S_{i}\right)=R\left(S_{\mathrm{i}}\right)$ [13], then the risk factor $i$ is in the highest level, on the basis of this, all factors in the highest level are picked up. In the reachable matrix, getting rid of the column and row elements corresponding to the risk factors in the highest level, accordingly, other levels can be acquired in the same way.

\subsection{Constructing the hierarchical structure model}

After the distribution of each level, all factors have been put in the corresponding level. According to the adjacent matrix, the directed line segment is used to express the relation between risk factors in the same level or in the neighbouring level, the hierarchical structure of system is expressed by the digraph. Through the construction of hierarchical structure model, the relation of risk factors can be determined. The risk factors in the highest level represent the main risks influencing the power grid investment, and the risk factors in the lowest level represent the risk source influencing the power grid investment, the risk factors in the middle levels form the risk chain to finish the conversion and transmit of risk factors in the highest level to risk factors in the lowest level. Through the construction of hierarchical structure model, the interaction between risk factors can be explained. 


\section{Case study}

As an example of Hunan power grid, based on the established risk index system, through the data analysis of Hunan power grid, the correlation matrix $R$ of each two risk factors is listed as Table. 2 .

Table 2. Correlation matrix.

\begin{tabular}{ccccccccccc}
\hline & A1 & A2 & A3 & B1 & B2 & B3 & C1 & C2 & C3 & C4 \\
\hline A1 & 1.00 & 0.54 & 0.41 & -0.63 & -0.88 & -0.41 & -0.85 & 0.67 & 0.88 & 0.82 \\
$\mathbf{A 2}$ & 0.54 & 1.00 & -0.45 & -0.91 & -0.16 & 0.53 & -0.11 & -0.12 & 0.52 & 0.03 \\
A3 & 0.41 & -0.45 & 1.00 & 0.39 & -0.78 & -0.95 & -0.75 & 0.93 & 0.26 & 0.86 \\
B1 & -0.63 & -0.91 & 0.39 & 1.00 & 0.27 & -0.11 & 0.24 & 0.05 & -0.44 & -0.31 \\
B2 & -0.88 & -0.16 & -0.78 & 0.27 & 1.00 & 0.77 & 0.87 & -0.94 & -0.55 & -0.72 \\
B3 & -0.41 & 0.53 & -0.95 & -0.11 & 0.77 & 1.00 & 0.70 & -0.87 & -0.17 & -0.55 \\
C1 & -0.85 & -0.11 & -0.75 & 0.24 & 0.87 & 0.70 & 1.00 & -0.88 & -0.19 & -0.85 \\
C2 & 0.67 & -0.12 & 0.93 & 0.05 & -0.94 & -0.87 & -0.88 & 1.00 & 0.48 & 0.96 \\
C3 & 0.88 & 0.52 & 0.26 & -0.44 & -0.55 & -0.17 & -0.19 & 0.48 & 1.00 & 0.55 \\
C4 & 0.82 & 0.03 & 0.86 & -0.31 & -0.72 & -0.55 & -0.85 & 0.96 & 0.55 & 1.00 \\
\hline
\end{tabular}

Based on the SPSS, the correlation matrix between each two risk factors is calculated, after the conversion of correlation matrix by choosing the proper membership function, the correlation strength matrix is acquired, and the experimental analysis is adopted to determine the threshold, and the value of $\lambda$ is 0.07 , therefore, the corresponding adjacent matrix $A$ is listed as Table. 3 .

Table 3. Adjacent matrix.

\begin{tabular}{ccccccccccc}
\hline & A1 & A2 & A3 & B1 & B2 & B3 & C1 & C2 & C3 & C4 \\
\hline A1 & 1 & 0 & 0 & 1 & 1 & 0 & 1 & 0 & 1 & 1 \\
A2 & 0 & 1 & 0 & 1 & 0 & 0 & 0 & 0 & 1 & 0 \\
A3 & 0 & 0 & 1 & 0 & 1 & 1 & 1 & 1 & 0 & 1 \\
B1 & 0 & 0 & 0 & 1 & 0 & 0 & 0 & 0 & 0 & 0 \\
B2 & 0 & 0 & 0 & 0 & 1 & 1 & 1 & 1 & 0 & 0 \\
B3 & 0 & 0 & 0 & 0 & 0 & 1 & 1 & 1 & 0 & 0 \\
C1 & 0 & 0 & 0 & 0 & 0 & 0 & 1 & 1 & 0 & 1 \\
C2 & 0 & 0 & 0 & 0 & 0 & 0 & 0 & 1 & 0 & 1 \\
C3 & 0 & 0 & 0 & 0 & 0 & 0 & 0 & 0 & 1 & 0 \\
C4 & 0 & 0 & 0 & 0 & 0 & 0 & 0 & 0 & 0 & 1 \\
\hline
\end{tabular}

According to the acquired adjacent matrix, the reachable matrix is calculated as Table. 4.

Table 4. Reachable matrix.

\begin{tabular}{ccccccccccc}
\hline & A1 & A2 & A3 & B1 & B2 & B3 & C1 & C2 & C3 & C4 \\
\hline A1 & 1 & 0 & 0 & 1 & 1 & 1 & 1 & 1 & 1 & 1 \\
A2 & 0 & 1 & 0 & 1 & 0 & 0 & 0 & 0 & 1 & 0 \\
A3 & 0 & 0 & 1 & 0 & 1 & 1 & 1 & 1 & 0 & 1 \\
B1 & 0 & 0 & 0 & 1 & 0 & 0 & 0 & 0 & 0 & 0 \\
B2 & 0 & 0 & 0 & 0 & 1 & 1 & 1 & 1 & 0 & 1 \\
B3 & 0 & 0 & 0 & 0 & 0 & 1 & 1 & 1 & 0 & 1 \\
C1 & 0 & 0 & 0 & 0 & 0 & 0 & 1 & 1 & 0 & 1 \\
C2 & 0 & 0 & 0 & 0 & 0 & 0 & 0 & 1 & 0 & 1 \\
C3 & 0 & 0 & 0 & 0 & 0 & 0 & 0 & 0 & 1 & 0 \\
C4 & 0 & 0 & 0 & 0 & 0 & 0 & 0 & 0 & 0 & 1 \\
\hline
\end{tabular}

According to the analysis above, the six-level hierarchical structure model is established, which can be seen as Figure. 1. 


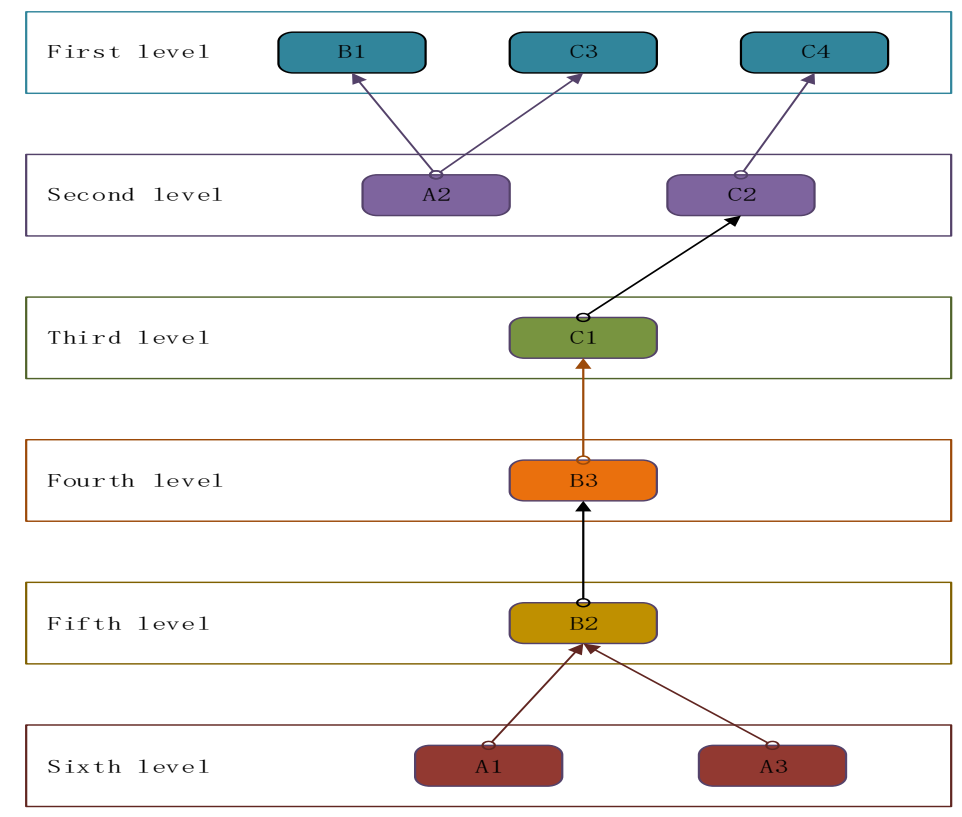

Figure 1. Graph of hierarchical structure model.

Through the established hierarchical structure model, it can be seen that the risk factors in the first level are B1, C3, C4, which represent the uncertainty of load demand, technological level and power quality of power grid investment respectively, the risk factors in the second level are A2, C2, which represent the average power price and grid structure, the risk factor in the third level is $\mathrm{C} 1$, which represents the power supply capacity, the risk factor in the fourth level is $\mathrm{B} 3$, which represents the loan interest rate, the risk factor in the fifth level is B2, which represents the GDP growth, the risk factors in the sixth level are A1, A3, which represent the electricity purchasing cost and the new energy policy.

According to the Figure 1, it can be seen that the uncertainty of load demand, technological level and power quality are in the top level, which shows that they are the direct and key risk factors influencing the power grid investment, and the electricity purchasing cost and the new energy policy are in the bottom level, which shows that they are the basic and key risk factors, the A1 and $\mathrm{A} 3$ influence the $\mathrm{B} 2$ directly, which is the risk factor in the fifth level, B2 influences the B3 directly, which is in the fourth level, B3 influences the $\mathrm{C} 1$ directly, which is in the third level, $\mathrm{C} 1$ influences the $\mathrm{C} 2$ directly, which is in the second level, C2 influences the C4 directly, which is in the top level, and A2 in the second level influences the B1 and C3 directly, which are in the top level. Through the 6-level hierarchical structure model, it forms the interaction process of risks from the original risks to the direct and key risks. Based on the process, by avoiding the production of risks in the bottom level and blocking the spread of risks in the middle level, the influence of risks can be decreased in the process of origin and spread.

\section{6 conclusion}

The interpretative structural model based on SPSS proposed in this paper, taking the strength of risk coupling between different risks into consideration, and achieving the conversion of interaction between factors from qualitative analysis to quantitative analysis by the application of the correlation analysis, which decreases the subjectivity of the expert scoring method and ensures the rationality and accuracy of the analysis results. The established hierarchical structure model reflects the mechanisms of interaction between risk factors, which lays the foundation for the decoupling and dimensionality reduction of risk factors and provides the basis for risk quantification and risk protection.

\section{Acknowledgments}

The authors acknowledge the support by the state grid science and technology projects (Grant: SGSHJY00GPJS1800060).

\section{References}

1. E. O. Crousillat, P. Dorfner, P. Alvarado, and H. M. Merrill. (1993) Conflicting objectives and risk in power system planning. IEEE Trans. Power Syst. 8(3): 887-893.

2. R. Mark Rylatt, J. Richard Snape, Peter Allen. (2015) Exploring Smart Grid Possibilities: A Complex Systems Modelling Approach. J. Smart Grid. pp. 115 .

3. M. Wang, Z. Tan, and R. Zhang. (2006) Risk Evaluation Model of the Power Grid Investment Based on Increment Principle. Trans. China Electrotech. Soc., 21(9): 18-24. 
4. D. F. Chen, F. Luo, Y. Feng. (2013) Analysis of Flight Safety Risk Coupling Based on Fuzzy Sets and Complex Network. 2013 International Conference on Management Science \& Engineering. pp. 329-334.

5. Y. M. Han, Z. Q. Geng, Q. X. Zhu, X. Y. Lin. (2015) Energy consumption hierarchical analysis based on interpretative structural model for ethylene production. Chinese Journal of Chemical Engineering. pp. 2029-2036.

6. Y. L. Chen, Z. Y. Zhao. (2018) Study on the Improved Interpretive Structural Modeling of the Factors Influence the Development of Regional Renewable Energy Power Generation Projects. Power System and Clean Energy. 34(2): 1-8.

7. I. B. Turksen. (2004) Ontological grounding of fuzzy theory. 2004 IEEE International Conference on Fuzzy Systems. pp. 1-6.

8. L. M. Ma, D. F. Li, H. X. Guo. (2015) Application of optimized BP neural network based on genetic algorithm in crude oil production prediction: a case study of BED test area in Daqing Oilfield. Pract. Cognition Math. 45(24): 117-128.

9. Y. F. Zhong, Y. T. Mei, B. B.Wu, D. Chen. (2012) Application of BP Neural Network Based on GA Optimization. Uplift Pressure Forecasting Dam. 30(6): 98-101.

10. L. L. Song, C. H. Deng, Q. S. Xu, F. F. Zeng, J. F. Huang. (2012) The operating efficiency evaluation of power equipment based on correlation analysis. Electric Power. 45(8): 85-90.

11. M. Mo, L. Z. Zhao, Y. Gong, and Y.Wu. (2018) Research and application of BP neural network based on genetic algorithm optimization. Mod. Electron.Technique. 41(9): 41-44.

12. X. Q. Zhang, Y. L. Yuan, Z. C. Sun. (2017) Improved interpretive structural model of influencing factors of resident travel cost. Journal of Chongqing Jiaotong University. 36(7): 101-105.

13. Y. G. Wang, Y. Z. Wang. (2012) Study on interaction law of risk factors in airlines operating. Journal of Safety Science and Technology. 8(5): 111-115. 